\title{
Chemopreventive efficacy of Targretin in rodent models of urinary bladder, colon/intestine, head and neck and mammary cancers
}

\author{
RONALD A. LUBET ${ }^{1}$, MARGIE L. CLAPPER ${ }^{2}$, DAVID L. MCCORMICK ${ }^{3}$, \\ MICHAELA. PEREIRA ${ }^{4}$, W-C. L. CHANG ${ }^{2}$, VERNON E. STEELE ${ }^{1}$, SUSAN M. FISCHER $^{5}$, \\ M. MARGARET JULIANA ${ }^{6}$ and CLINTON J. GRUBBS ${ }^{6}$ \\ ${ }^{1}$ Division of Cancer Prevention, National Cancer Institute, Bethesda, MD; ${ }^{2}$ Division of Population \\ Science, Fox Chase Cancer Center, Philadelphia, PA; ${ }^{3}$ Life Sciences Operation, IIT Institute, Chicago, IL; \\ ${ }^{4}$ Ohio State University, Columbus, OH; ${ }^{5}$ University of Texas, M.D. Anderson Cancer Center, Houston, TX; \\ ${ }^{6}$ Departments of Genetics and Surgery, University of Alabama at Birmingham, Birmingham, AL, USA
}

Received November 11, 2011; Accepted December 12, 2011

DOI: 10.3892/or.2012.1673

\begin{abstract}
The chemopreventive efficacy of Targretin was evaluated in various rodent cancer models. In the rat model of 4-hydroxybutyl(butyl)nitrosamine (OH-BBN)-induced urinary bladder cancer, it was found that Targretin administered in the diet (beginning one week after the last OH-BBN treatment) for 5.5 months increased the number and size of urinary bladder cancers. In the azoxymethane (AOM)-induced model of colon carcinogenesis (in which rats develop minimally invasive colonic cancers), Targretin was ineffective as a chemopreventive agent, decreasing neither tumor incidence nor multiplicity. Treatment of Min mice with Targretin for 45 days similarly failed to decrease the multiplicity of small intestinal tumors. Similarly, no preventive efficacy was noted for Targretin when the incidence of tumors in the head and neck model (squamous cell tongue tumors) induced by 4-nitroquinoline 1-oxide (4-NQO) were examined. In contrast, use of even a suboptimal dose of Targretin $(40 \mathrm{ppm})$ in a sensitive breast cancer model [methylnitrosourea (MNU)-induced $\mathrm{ER}^{+}$mammary cancers] reduced cancer multiplicity by $60 \%$. Finally, based on the hypothesis that Targretin may decrease the expression of COX-2, the effects of Targretin and COX inhibitors were compared in these models. There was minimal overlap of efficacy. That is, models which were relatively susceptible to NSAIDs or COX-2 inhibitors tended not to be sensitive to Targretin and vice versa.
\end{abstract}

\section{Introduction}

The RXR agonist Targretin interacts with a group of nuclear receptors designated RXR receptors which are present in

Correspondence to: Dr Ronald A. Lubet, National Cancer Institute, Executive Plaza North, Suite 2110, 6130 Executive Boulevard, Bethesda, MD 20852, USA

E-mai: lubetr@mail.nih.gov

Key words: cancer, Targretin, animal models, chemoprevention most cells in the body. These RXR receptors can then interact with and form heterodimers with the widest range of nuclear receptors (PPAR, CAR, PXR, RARs, TXR and VDR) (1). The resulting heterodimers initiate the activation of a wide variety of genes. Because of the wide range of physiological changes induced by RXR agonists, it was initially anticipated that they would prove useful as a generalized cancer prevention strategy (2). This hope was further supported by the fact that the retinoids (all-trans-retinoic acid, 13-cis-retinoic acid) which bind to RARs and in turn form heterodimers with RXRs, are active against a number of cancers including acute promyelocytic leukemia and squamous cell cancers of both the head and neck and skin $(3,4)$. However, this class of agents was limited by certain toxicities associated with RAR activation; including cutaneous toxicities. Early studies with the RXR agonist Targretin showed it to be highly active in a variety of cell lines in culture (5). Although in vivo experiments with this class of agents are more limited, Targretin and other RXR agonists have been shown to be highly effective in preventing both $\mathrm{ER}^{+}$and $\mathrm{ER}^{-}$mammary tumors in animal models $(6,7)$. With respect to the ER positive tumors, Targretin proved to be not only a preventive agent but was a highly effective therapeutic agent as well (6). Our laboratory, and others, has subsequently found that Targretin is active in mouse models of lung adenocarcinomas (8), as well as mouse models of squamous cell and small cell cancer of the lung (9). When taken together, these data suggest that Targretin might be useful in a wide variety of cancers. Furthermore, there have been review articles presented in the general literature which propose that the RXR agonists may be active in a variety of cancers (10).

In the present study, we aimed to expand the evaluation of the efficacy of Targretin to additional models of carcinogenesis. The urinary bladder (11), head and neck (12), colon/ intestine $(13,14)$ and mammary $(6)$ models have been employed previously to examine the efficacy of a variety of potential chemopreventive agents. Interestingly, it was proposed recently that some of the efficacy of the RXR agonists in the mammary gland may be due to inhibition of COX-2 expression 
Table I. Effect of Targretin on hydroxybutyl(butyl)nitrosamine (OH-BBN)-induced urinary bladder lesions in female Fischer-344 rats.

\begin{tabular}{l} 
Group \\
\cline { 3 - 5 } Carcinogen
\end{tabular}

by the RXR agonists (15). Based on the present results and our previously published results, we compared the efficacy obtained with COX inhibitors (NSAIDs or celecoxib) to those obtained with Targretin. The data suggest that COX inhibitors and Targretin are unlikely to function by similar mechanisms.

\section{Materials and methods}

Chemicals. Targretin (bexarotene, LDG1069) was obtained from the Division of Cancer Prevention, Chemical Repository, National Cancer Institute.

Urinary bladder cancer model. This model has been previously described $(11,16)$. The carcinogen hydroxybutyl(butyl)nitrosamine $(\mathrm{OH}-\mathrm{BBN})$ was purchased from TCI America (Portland, OR). OH-BBN (150 mg/gavage) was administered twice a week for 8 weeks beginning when the female Fischer-344 rats were 56 days of age. The carcinogen was administered $(1.0 \mathrm{ml}$ by gavage) in ethanol:water $(25: 75, \mathrm{v} / \mathrm{v})$. One week following the last dose of $\mathrm{OH}-\mathrm{BBN}$, the animals received Targretin supplemented in the diet (Teklad, 250 or $100 \mathrm{ppm}$ ). The mash diet-Targretin mixture was prepared using a Patterson-Kelly blender with an intensifier bar. The rats received this diet until the end of the study (for 5.5 months). Animals were observed daily, weighed weekly, and palpated for urinary bladder masses twice a week. Rats were sacrificed when they developed a large palpable bladder lesion or were observed to have bloody urine. At necropsy, urinary bladders were inflated with $10 \%$ buffered formalin. After fixation, the bladder was observed under a high intensity light for gross lesions. Each lesion was dissected and processed for pathological classification. The multiplicity and weight of the urinary bladder tumors were determined at the end of the study. Statistical analysis of bladder weights was performed by the Wilcoxon rank test and the final cancer incidence by the Fischer's exact test.

AOM colon cancer model. AOM was purchased from Sigma Chemical Co. (St. Louis, MO) and the AIN-76A diet was purchased from Dyets Inc. (Bethlehem, PA). Male Fischer-344 rats were obtained from Charles River Laboratories (Frederick, MD) at 5-6 weeks of age. The rats received AIN-76A diet upon arrival. One week later, the rats were administered $15 \mathrm{mg} / \mathrm{kg}$
BW AOM once a week for three consecutive weeks (13). Five weeks later, the rats were randomly assigned to the following three treatment groups of 40 animals each: 0, 50 and $150 \mathrm{ppm}$ Targretin in their diet. The rats were sacrificed at 44 weeks after the first injection of AOM. At necropsy, the colons were excised, cut along the longitudinal axis, flushed with cold saline, and evaluated for tumors. The tumors were fixed in $10 \%$ buffered formalin, embedded in paraffin, and stained with hematoxylin and eosin for histopathological evaluation. Colon tumor multiplicity was analyzed by an ANOVA followed by the Bonferroni t-test, using a P-value $<0.05$.

Intestinal tumors in Min mice. Male Apc $\mathrm{Apin}^{+\mathrm{FCCC}}$ mice (8-weeks old) were obtained from the Laboratory Animal Facility at the Fox Chase Cancer Center (FCCC) and were acclimated to a modified AIN-76A semi-purified diet for one week prior to starting treatment with Targretin (14). At 9 weeks of age, mice were randomized to receive control diet or diet supplemented with 400 or 600 ppm of Targretin $(\mathrm{N}=10$ / group). The experimental diets were prepared in the laboratory biweekly and stored at $-20^{\circ} \mathrm{C}$. Body weights were recorded weekly to monitor toxicity. Animals were maintained on the experimental diets ad libitum for 45 days.

Mice were sacrificed at the end of the experiment. Gross lesions were counted and recorded. Adenomas were defined as circumscribed neoplasms composed of tubular and/or villous structures and lined with dysplastic epithelium. Cancers had to meet three criteria: i) invasion into at least the submucosa; ii) eliciting a desmoplastic reaction; and iii) exhibiting cytological features of neoplasia. Body weights and tumor multiplicities were compared among treatment groups using the Wilcoxon Rank Sum test. The P-values of the pairwise comparisons were adjusted using the Bonferroni multiple comparisons.

Squamous cancers of the head and neck. Male Fischer-344 rats were obtained from the NCI Frederick animal facility. Beginning at 8 weeks of age, rats were exposed to drinking water containing 20 ppm 4-NQO (Aldrich Chemical Company, Milwaukee, WI) for a period of 10 weeks. Treatment with Targretin was initiated one day after the final administration of 4-NQO. Animals were monitored daily and weighed twice a week. The rats were sacrificed if they lost weight for 


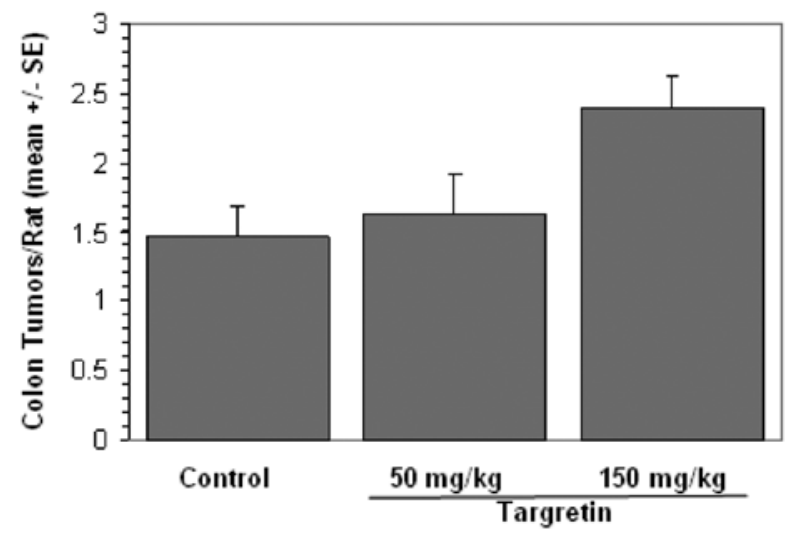

Figure 1. Effect of Targretin on AOM-induced colon tumors. Male Fischer-344 rats (5-6-weeks old) were administered $15 \mathrm{mg} / \mathrm{kg}$ BW AOM once a week for three consecutive weeks. Five weeks later, the rats (40 per group) were randomly assigned to treatment groups receiving: 0,50 or $150 \mathrm{ppm}$ Targretin in their diet. The rats were sacrificed at 44 weeks after the first injection of $\mathrm{AOM}$ and tumor counts determined as previously described (13).

two successive weeks. The remaining rats were sacrificed 18 weeks following the last administration for 4-NQO. All rats underwent a complete necropsy that focused on the tongue, oral cavity and gastrointestinal tract. More specific details regarding the assay are presented by McCormick et al (12).

Mammary cancer model. The carcinogen methylnitrosourea (MNU) was obtained from the National Cancer Institute Chemical Repository. Female Sprague-Dawley rats were obtained from Harlan Laboratory, Inc. (Indianapolis, IN), and were administered the carcinogen $(75 \mathrm{mg} / \mathrm{kg} \mathrm{BW})$ by IV injection at 50 days of age (6). Five days after MNU, the animals received a diet (Teklad) supplemented with Targretin (40 ppm). The animals received this diet until termination of the study at 126 days after MNU. The rats were weighed once a week, palpated for mammary tumors twice a week, and observed daily for signs of toxicity. Mammary tumors were excised, weighed, and processed for histological classification at termination of the study. Differences in cancer multiplicity were determined by the Armitage test.

Animal use approvals. All animal experiments were conducted in facilities at the Fox Chase Cancer Center, IIT Research Institute, Ohio State University, and the University of Alabama at Birmingham following the procedures approved by the Institutional Animal Care and Use Committee of the respective institutions.

\section{Results}

$O H$-BBN-induced urinary bladdercancer. One week following the last administration of $\mathrm{OH}-\mathrm{BBN}$, the animals were administered Targretin in the diet (at either 250 or $100 \mathrm{ppm}$ ). The rats were observed for urinary bladder tumors for an additional 5.5 months. Based on palpable urinary bladder masses, tumors developed more rapidly in those rats receiving Targretin. This was confirmed at termination of the study when the size of the urinary bladder plus lesions was significantly larger in the Targretin-treated rats. As indicated in Table I, the weights of
Table II. Multiplicity of small intestinal lesions in FCCC $\mathrm{Apc}^{\mathrm{Min}}$ mice receiving Targretin ${ }^{\mathrm{a}}$.

\begin{tabular}{lrc}
\hline Group & N & $\begin{array}{c}\text { Multiplicity of histologically } \\
\text { confirmed lesions } \\
\text { (mean } \pm \text { SEM) }\end{array}$ \\
\hline Control & 10 & $10.2 \pm 2.1$ \\
400 ppm Targretin & 9 & $20.1 \pm 5.2$ \\
600 ppm Targretin & 10 & $20.9 \pm 7.8$ \\
\hline
\end{tabular}

Targretin was administered in powdered AIN-76A diet. ${ }^{b}$ No significant difference was detected among treatment groups using the Wilcoxon Rank Sum test $(\mathrm{P}=0.134)$.

the bladder lesions were 97 and 50\% greater than the controls at the high and low doses of Targretin, respectively. After histological classification, an increase in the number of transitional cell carcinomas was also observed in the rats receiving Targretin at the low dose.

Colon cancer in rats. The number of colon lesions observed in rats in each treatment group is summarized in Fig. 1. No significant difference was observed in the number of colon cancers in Targretin-treated vs. control animals. In fact, the mean multiplicity of colonic tumors was higher in the treated group, although not significantly, as compared to untreated controls.

Intestinal tumors in MIN mice. The survival rate for mice receiving diets supplemented with 0 ppm (control), 400 and $600 \mathrm{ppm}$ Targretin was $\geq 90 \%$. While a high rate of survival was observed among animals administered Targretin, the body weights of Targretin-treated animals remained stable while those of control animals increased gradually over time as anticipated. The body weights of animals administered Targretin at 400 and 600 ppm were significantly decreased relative to controls at the end of the study ( $\mathrm{P} \leq 0.01$ by the Wilcoxon Rank Sum test).

The number of small intestinal lesions observed upon histopathological evaluation of mice in each treatment group is summarized in Table II. No significant difference was observed in the number of small intestinal lesions present in Targretin-treated vs. control animals $(\mathrm{P}=0.134)$ by the Wilcoxon Rank Sum test). In fact, the mean multiplicity of small intestinal tumors was higher in both treated groups, although not significantly, as compared to untreated controls.

Head and neck cancers. Results in the head and neck model (Table III) showed that Targretin (100 or $250 \mathrm{ppm}$ ) administered beginning at week 10 or week 16 , failed to decrease either the incidence or degree of invasion of squamous cell cancers of the tongue.

Mammary cancers. In this mammary model, the cancers induced by MNU are $\mathrm{ER}^{+}$. As shown in Fig. 2, in the carcinogen-treated only group mammary cancers became palpable at 35-40 days after MNU, and increased rapidly throughout the 
Table III. Influence of Targretin on the incidence and invasiveness of NQO-induced squamous cell carcinoma of the oral cavity in Fischer-344 rats.

A, Influence of Targretin on the incidence of squamous cell carcinoma of the oral cavity

Lesion no. (\% incidence)

\begin{tabular}{|c|c|c|c|c|c|}
\hline Agent & $\begin{array}{l}\text { Agent dose } \\
\text { (mg/kg diet) }\end{array}$ & $\begin{array}{l}\text { Exposure schedule } \\
\text { (weeks) }\end{array}$ & $\begin{array}{l}\text { Squamous epithelial } \\
\text { hyperplasia }\end{array}$ & $\begin{array}{l}\text { Squamous cell } \\
\text { papilloma }\end{array}$ & $\begin{array}{l}\text { Squamous } \\
\text { cell carcinoma }\end{array}$ \\
\hline None (control) & 0 & - & 4/30 (13) & $1 / 30(3)$ & 25/30 (83) \\
\hline Targretin & 100 & $10-24$ & $2 / 29(7)$ & $0 / 29(0)$ & $26 / 29(90)$ \\
\hline Targretin & 250 & $10-24$ & $3 / 28$ (11) & 0/28 (0) & $24 / 28(86)$ \\
\hline Targretin (late) & 100 & $16-24$ & $2 / 30(7)$ & 2/30 (7) & $26 / 30(87)$ \\
\hline Targretin (late) & 250 & $16-24$ & $5 / 30(17)$ & 2/30 (7) & $23 / 30(77)$ \\
\hline
\end{tabular}

B, Influence of Targretin on the invasiveness of squamous cell carcinoma of the oral cavity

\begin{tabular}{|c|c|c|c|c|c|}
\hline \multirow[b]{2}{*}{ Agent } & \multirow{2}{*}{$\begin{array}{l}\text { Agent dose } \\
\text { (mg/kg diet) }\end{array}$} & \multirow{2}{*}{$\begin{array}{l}\text { Exposure schedule } \\
\text { (weeks) }\end{array}$} & \multicolumn{3}{|c|}{ Incidence according to the invasion score ${ }^{a}$} \\
\hline & & & +1 & +2 & +3 \\
\hline None (control) & 0 & - & $2 / 30(7)$ & $2 / 30(7)$ & $21 / 30(70)$ \\
\hline Targretin & 100 & $10-24$ & $1 / 29(3)$ & 4/29 (14) & $21 / 29(72)$ \\
\hline Targretin & 250 & $10-24$ & $1 / 28(4)$ & $2 / 28(7)$ & $21 / 28(75)$ \\
\hline Targretin (late) & 100 & $16-24$ & $1 / 30(3)$ & 1/30 (3) & $24 / 30(80)$ \\
\hline Targretin (late) & 250 & $16-24$ & $1 / 30(3)$ & $5 / 30(17)$ & $17 / 30(57)$ \\
\hline
\end{tabular}

anvasion score described in reference 12 .

remainder of the study. The administration of even a suboptimal dose of Targretin (40 ppm) greatly delayed the time of appearance of the mammary cancers. At the end of the study, the number of mammary cancers was decreased by Targretin from 5.2 tumors/rat in the controls to 2.7 (a 52\% decrease). A significant decrease $(61 \%)$ in the weight of the mammary cancers was also observed. Thus, based on latency period, multiplicity, and cancer weight, Targretin at this relatively low dose was highly effective in preventing $\mathrm{ER}^{+}$mammary cancers.

Comparison of the efficacy of NSAIDs/celecoxib and Targretin in various models. In Table IV, we have compared the relative efficacy of NSAIDs, celecoxib or Targretin for their ability to inhibit cancer formation in various models. The data on Targretin are based on the experiments in this study and on previously published studies performed by the authors. The data on the NSAIDs and celecoxib is from a previously published review study by the authors which itself references the various primary studies. The major finding is that there is minimal overlap between efficacy of Targretin and the various COX1/2 inhibitors (NSAIDs and celecoxib). Thus, Targretin was highly effective in models of breast cancer, whereas NSAIDs showed no activity and celecoxib showed limited (albeit occasionally significant) activity. In contrast, the NSAIDs and Coxibs were highly effective in models of urinary bladder, colon/intestine, and head and neck tumors,
Table IV. Relative efficacy of Targretin vs. NSAIDs or celecoxib to prevent tumor formation in various rodent models.

\begin{tabular}{lccc}
\hline & \multicolumn{3}{c}{ Efficacy of agents } \\
\cline { 2 - 4 } Cancer model & NSAIDs $^{\mathrm{a}}$ & Celecoxib $^{\mathrm{a}}$ Targretin $^{\mathrm{b}}$ \\
\hline Urinary bladder (rat) & ++++ & ++++ & - \\
AOM colon (rat) & ++++ & ++++ & - \\
Min intestine (mouse) & +++ & +++ & - \\
Head and neck (rat) & +++ & +++ & - \\
ER $^{+}$mammary (rat) & $+/-$ & + & ++++ \\
ER mammary (mouse) & - & + & +++ \\
Lung adenocarcinoma (mouse) & - & - & +++
\end{tabular}

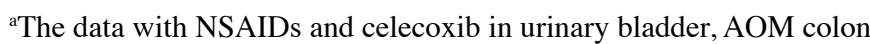
and Min intestine are based on a recent report (27). The data on the head and neck model are from ref. 12, and that on $\mathrm{ER}^{+}$positive mammary cancer from ref. 28. Data on ER mammary cancer and lung cancer are from unpublished data. ${ }^{\mathrm{b}}$ The data for Targretin on urinary bladder, AOM colon, Min intestine and head and neck cancer are from the present study. Data on $\mathrm{ER}^{+}$and $\mathrm{ER}^{-}$mammary cancer are from refs. 6, 7 and 19 and that on lung adenocarcinomas from refs. 8, 9 and 20.

while Targretin was ineffective in all these models. Thus, there are three points to be made regarding the data: i) there appears to be minimal overlap between the two classes of agents 


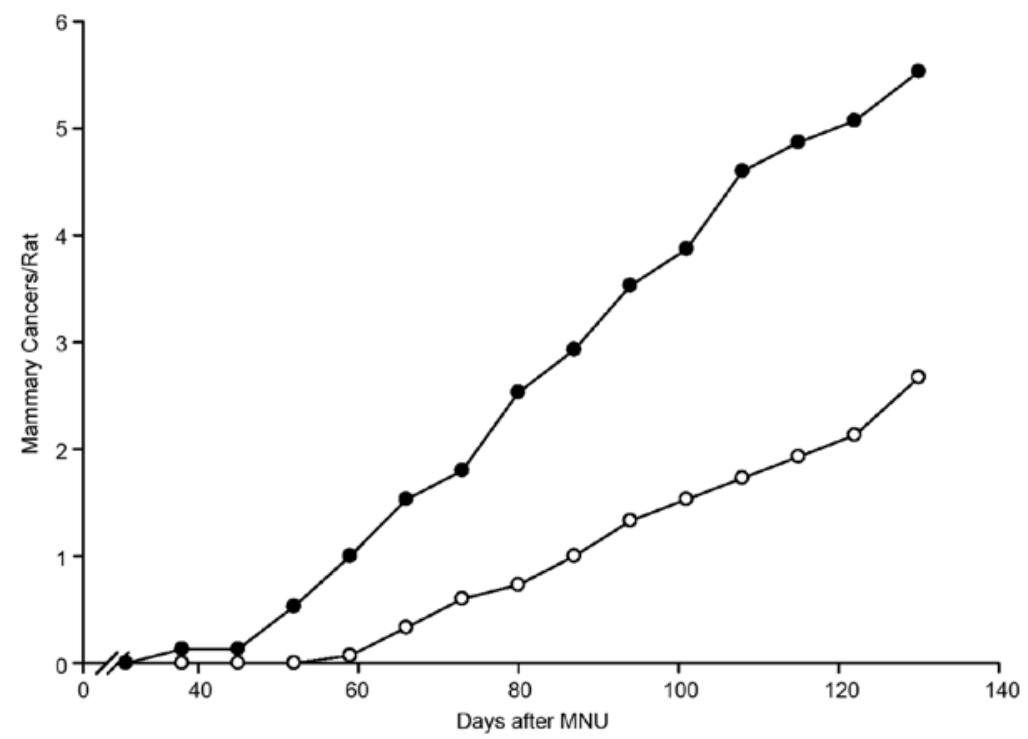

Figure 2. Effect of Targretin on MNU-induced mammary cancers. MNU was administered at 50 days of age. Diet supplementation with Targretin (40 ppm diet) was started 5 days later, and continued until the end of the study. The groups were: o, Targretin; $\bullet$, controls.

(Targretin vs. NSAIDs/celecoxib); ii) there is a close correlation between NSAIDs and celecoxib; iii) it shows clearly that no single compound or class of compounds is likely to be highly effective against the widest range of cancers.

\section{Discussion}

Targretin is the only clinically approved RXR agonist for cancer, and has been employed in the treatment of both cutaneous lymphomas and NSCLC $(17,18)$. The RXR receptors form heterodimers with a variety of nuclear receptors (PPARs, CAR, LXR, RARs, VDR and TXR) (1). Although they form heterodimers with the VDR, RARs and the thyroxin receptor, they form permissive heterodimers with many other receptors including PPARs, CAR and LXR. Permissive heterodimers respond both to ligands or agonists for the cognate receptor (e.g., rosiglitazone/PPAR $\gamma$ ) as well as RXR agonists. Thus, exposure to the RXR agonists alone induces some of the same responses as those observed following addition of ligands/agonists for the cognate receptor. Presumably this is accomplished by interaction with an endogenous agonist for the respective receptor. Treatment of rats with RXR agonists causes many of the same effects in the liver as treatment with PPAR $\alpha$ agonists or CAR agonists (19). Since RXRs form permissive heterodimers with a wide variety of receptors and their functions are purported to be involved in a wide variety of cancers, it implies that the RXR agonists may be effective preventive agents in a wide variety of cancers (10).

In preclinical chemopreventive studies, Targretin and certain other RXR agonists have proven to be profoundly effective in preventing $\mathrm{ER}^{+}$tumors in rats and ER mammary tumors in transgenic mice. In fact, this efficacy is not limited to Targretin but, as we have demonstrated, extends to a variety of RXR agonists in that model (20). As can readily be seen (Fig. 2), Targretin at a suboptimal dose of $40 \mathrm{ppm}$ increased the latency and decreased the multiplicity of mammary cancers. Our laboratory, and more recently others, have shown that Targretin was effective in blocking formation of lung adenomas and adenocarcinomas in mice $(8,21)$. More recently, we have found Targretin to be profoundly effective in prevention of small cell lung cancer in a transgenic model in which both p53 and Rb are knocked out (9). Thus, in at least two organ sites, Targretin appeared to be highly effective. We wished to expand this investigation to a number of other organ sites and models which are commonly used in preclinical prevention studies. The models examined included: i) $\mathrm{OH}-\mathrm{BBN}$ induced invasive bladder cancers in rats; ii) 4-NQO induced squamous cells cancers of the tongue in rats; iii) AOM induced minimally invasive colon cancers in rats; iv) and intestinal tumors that arise spontaneously in Min mice (which bear a germline APC mutation). The $\mathrm{OH}-\mathrm{BBN}$ induced urinary bladder cancers of the rat show many of the gene and protein expression changes associated with human urinary bladder cancer; e.g., increased levels of COX-2, survivin, cyclin D1, EGFR, and decreased levels of FHIT (22). The tumors have similarities to invasive bladder cancer in humans as examined by array analyses (23). The AOM model in rats and the Min model in mice both have alterations in the Wnt pathway (APC and $\beta$-cateinin) and have significant overlap; as determined by microarrays with human colon cancer which similarly is driven by the same alteration (APC mutations) (24). Finally, the squamous cell carcinomas of the tongue appear histologically similar to human cancers of the head and neck (13). However, there have been more limited molecular comparisons. All of these models have been used to screen for potential chemopreventive agents.

$\mathrm{OH}-\mathrm{BBN}$ is an organ specific carcinogen that induces urinary bladder cancers both in mice and rats. We have previously shown that a variety of agents are highly effective (naproxen, celcecoxib, Iressa and lapatinib) when initiated in this model late in tumor development (16). Surprisingly, Targretin significantly increased the incidence and size of tumors that developed in this model; as shown in Table I. These 
data are similar to our recent finding that the PPAR $\gamma$ agonist rosiglitazone promotes urinary bladder cancer formation in this model (25). As mentioned above, RXR agonists may have many similar physiologic effects as the administration of PPAR or CAR agonists. Thus, the effect may be mediated by the PPAR $\gamma$ heterodimer formation.

Targretin was evaluated in the 4-NQO induced model of head and neck cancer in rats for preventive activity. The tumors appear histologically similar to more advanced cancers of the head and neck in humans (12). It was observed that Targretin ( 250 or $100 \mathrm{ppm}$ ) failed to decrease both the incidence of invasive tumors of the tongue and the relative degree of invasion.

The preventive effects of Targretin were also evaluated in two models of colon/intestinal cancer; the rat AOM induced model and the Min mouse model. Min mice have a germ line mutation in the APC gene; thus, serving as a model of intestinal carcinogenesis. Min mice develop multiple small intestinal tumors, but also develop a limited number of colonic tumors. This model has proven to be highly responsive to COX-2 inhibitors and ornithine decarboxylase (ODC) inhibitors (26). As shown in Table II, Targretin failed to inhibit the formation of intestinal tumors, and in fact may have slightly increased the formation of these lesions. The other model employs the organ specific carcinogen AOM. The resulting tumors routinely develop mutations in the $\mathrm{APC} / \beta$-cateinin pathway and, thus, are similar to human cancers. In both cases, chemopreventive efficacy with Targretin was not observed.

Overall, Targretin (and possibly other RXR agonists) was ineffective in a variety of relatively standard rodent tumor models. This includes urinary bladder, colon/intestine, and head and neck. In contrast, this agent was highly effective as a preventive agent in $\mathrm{ER}^{+}$and $\mathrm{ER}^{-}$mammary cancers $(6,7)$ and has shown activity in animal models of lung cancer; including lung adenocarcinomas and small cell cancer of the lung $(8,9)$. However, there is one particularly interesting aspect of these results that warrants some comment. One of the controversies that arise regarding Targretin is the possible mechanism of action of this agent. It has been reported that Targretin can reduce the expression of COX-2 protein in normal mammary epithelium and tumors (15). The implication is that inhibition of COX-2, although perhaps indirectly, may be a significant target. In Table IV, we have compared the efficacy of Targretin and NSAIDs/COX-2 inhibitors in various animal models. As shown, there is virtually no overlap. Thus, Targretin is highly effective in various mammary models, while NSAIDs/ COX-2 inhibitors demonstrate limited activity. In contrast, both NSAIDs and celecoxib are profoundly effective in the urinary bladder (naproxen and celecoxib), AOM colon (celecoxib, naproxen and piroxicam), Min mouse intestine model (piroxicam, celecoxib, naproxen and sulindac), and in the head and neck model (piroxicam, celecoxib and NO-naproxen) (27). Targretin was relatively ineffective in all these models. One of the great difficulties in determining the mechanism of action of Targretin in various organs is that, unlike COX-2 inhibitors, Targretin interacts with different nuclear receptors in various organs and likely functions by different mechanisms in different target organs. The data in Table IV also re-emphasize the fact that no single agent or class of agents is likely to be a profoundly effective preventive agent for all types of epithelial cancers.

\section{Acknowledgements}

The authors wish to thank Jeanne Hale, Mary Jo Cagle, and Julie Gray for their secretarial and editorial services. Research materials for the studies were in part obtained by the NCI contract number HHSN261200433001C.

\section{References}

1. Bastien $\mathbf{J}$ and Rochette-Egly $\mathrm{C}$ : Nuclear retinoid receptors and the transcription of retinoid-target genes. Gene 328: 1-16, 2004.

2. Sporn MB and Suh N: Chemoprevention of cancer. Carcinogenesis 21: 525-530, 2000

3. Kraemer KH, DiGiovanna JJ, Moshell AN, Tarone RE and Peck GL: Prevention of skin cancer in xeroderma pigmentosum with the use of oral isotretinoin. N Engl J Med 318: 1633-1637, 1988.

4. Lo-Coco F and Ammatuna E: Front line clinical trials and minimal residual disease monitoring in acute promyelocytic leukemia. Curr Top Microbiol Immunol 313: 145-156, 2007.

5. Lu XP, Fanjul A, Picard N, et al: Novel retinoid-related molecules as apoptosis inducers and effective inhibitors of human lung cancer cell in vivo. Nature Med 3: 686-690, 1997.

6. Gottardis MM, Bischoff ED, Shirley MA, Wagoner MA, Lamph WW and Heyman RA: Chemoprevention of mammary carcinoma by LGD1069 (Targretin): an RXR selective ligand. Cancer Res 56: 5566-5570, 1996.

7. Wu K, Zhang $\mathrm{Y}, \mathrm{Xu} \mathrm{XC}$, et al: The retinoid $\mathrm{X}$ receptor-selective retinoid, LGD1069, prevents the development of estrogen receptor-negative mammary tumors in transgenic mice. Cancer Res 62: 6376-6380, 2002.

8. Wang Y, Zhang Z, Yao R, Jia D, Wang D, Lubet RA and You M: Prevention of lung cancer progression by bexarotene in mouse models. Oncogene 25: 1320-1329, 2006.

9. Wang Y, Wen W, Yi Y, Zhang Z, Lubet RA and You M: Preventive effects of bexarotene and budesonide in genetically engineered mouse model of small cell lung cancer. Cancer Prev Res 12: 1059-1064, 2009.

10. Liby KT, Yore MM and Sporn MB: Triterpenoids and rexinoids as multifunctional agents for the prevention and treatment of cancer. Nat Rev Cancer 7: 357-369, 2007.

11. Steele VE, Rao CV, Zhang Y, et al: Chemopreventive efficacy of naproxen and nitric-oxide naproxen in rodent models of colon, urinary bladder, and mammary cancers. Cancer Prev Res 2: 951-956, 2009.

12. McCormick DL, Phillips JM, Horn TL, Johnson WD, Steele VE and Lubet RA: Overexpression of cyclooxygenase-2 in rat oral cancers and prevention of oral carcinogenesis in rats by selective and non-selective COX inhibitors. Cancer Prev Res 3: 73-81, 2010.

13. Li H, Kramer PM, Lubet RA, Steele VE, Kelloff GJ and Pereira MA: Effect of calcium on azoxymethane-induced aberrant crypt foci and cell proliferation in the colon of rats. Cancer Lett 124: 39-46, 1998.

14. Cooper HS, Chang WC, Coudry R, et al: Generation of a unique strain of multiple intestinal neoplasia (Apc(+/Min-FCCC)) mice with a significantly increased numbers of colorectal adenomas. Mol Carcinog 44: 31-41, 2005.

15. Kong G, Kim H-T, Wu K, et al: The retinoid X receptor-selective retinoid, LGD1069, down-regulates cyclooxygenase-2 expression in human breast cells through transcription factor crosstalk: Implications for molecular-based chemoprevention. Cancer Res 65: 3462-3469, 2005.

16. Lubet RA, Steele VE, Juliana MM and Grubbs CJ: Screening agents for preventive efficacy in a bladder cancer model: study design, end points and gefitinib and naproxen efficacy. J Urol 183: 1598-1603, 2010.

17. Farol LT and Hymes KB: Bexarotene: a clinical review. Expert Rev Anticancer Ther 4: 180-188, 2004.

18. Dragnev KH, Ma T, Cyrus J, et al: Bexarotene plus erlotinib suppress lung carcinogenesis independent of KRAS mutations in two clinical trials and transgenic models. Cancer Prev Res 4: 818-826, 2011.

19. Wang Y, Yao R, Maciag A, Grubbs CJ, Lubet RA and You M: Organ-specific expression profiles of rat mammary gland, liver and lung tissues treated with Targretin, 9-cis retinoic acid, and 4-hydroxyphenylretinamide. Mol Cancer Ther 5: 1060-1072, 2006. 
20. Grubbs CJ, Lubet RA, Atigadda VR, et al: Efficacy of new retinoids in the prevention of mammary cancers and correlations with short-term biomarkers. Carcinogenesis 27: 1232-1239, 2006.

21. Liby K, Black CC, Royce DB, et al: The rexinoid LG 100268 and the synthetic triterpenoid CDDO-methyl amide are more potent than erlotinib for prevention of mouse lung carcinogenesis. Mol Cancer Ther 7: 1251-1257, 2008.

22. Yao R, Yi Y, Grubbs CJ, Lubet RA and You M: Gene expression profiling of chemically induced rat bladder tumors. Neoplasia 9: 207-221, 2007.

23. Williams PD, Lee JK and Theodorescu D: Molecular credentialing of rodent bladder carcinogenesis models. Neoplasia 10 838-846, 2008.

24. Kaiser S, Park YK, Franklin JL, Halberg RB, Yu M, Jessen WJ, Freudenberg J, Chen X, Haigis K, Jegga AG, Kong S, et al: Transcriptional recapitulation and subversion of embryonic colon development by mouse colon tumor models and human colon cancer. Genome Biol 8: R31, 2007.
25. Lubet RA, Fischer SM, Steele VE, Juliana MM, Desmond R and Grubbs CJ: Rosiglitazone, a PPAR gamma agonist: potent promoter of hydroxybutyl(butyl)nitrosamine-induced urinary bladder cancers. Int J Cancer 123: 2254-2259, 2008.

26. Jacoby RF, Cole CE, Tutsch K, Newton MA, Kelloff G, Hawk ET and Lubet RA: Chemopreventive efficacy of combined piroxicam and difluoromethylornithine treatment of Apc mutant Min mouse adenomas, and selective toxicity against Apc mutant embryos. Cancer Res 60: 1864-1870, 2000.

27. Fischer SM, Hawk E and Lubet RA: Non-steroidal anti-inflammatory drugs and coxibs in chemoprevention: a commentary based primarily on animal studies. Cancer Prev Res (Phila) 4: 1728-1735, 2011 .

28. Christov K, Grubbs CJ, Shilkaitis A, Juliana MM and Lubet RA: Short-term modulation of cell proliferation and apoptosis and preventive/therapeutic efficacy of various agents in a mammary cancer model. Clin Cancer Res 13: 5488-5496, 2007. 